MODELING, IDENTIFICATION AND CONTROL, 2004, VOL. 25, No. 4, 237-252

doi: $10.4173 /$ mic.2004.4.3

\title{
Development of an in vivo method for determining material properties of passive myocardium*
}

\author{
ESPEN W. REMME*, PETER J. HUNTER $\dagger$, OTTO SMISETH $\ddagger$, \\ CAREY STEVENS $\dagger$, STEIN INGE RABBEN $\ddagger$, HELGE \\ SKULSTAD $\ddagger$ and BJØRN ANGELSEN*
}

Keywords: Myocardium; material properties; estimation; finite clement modeling

Calculation of mechanical stresses and strains in the left ventricular (LV) myocardium by the finite element (FE) method relies on adequate knowledge of the material properties of myocardial tissue. In this paper we present a model-based estimation procedure to characterize the stress-strain relationship in passive $\mathbf{L V}$ myocardium. A 3D FE model of the LV myocardium was used, which included morphological fiber and sheet structure and a nonlinear orthotropic constitutive law with different stiffness in the fiber, sheet and sheet-normal directions. The estimation method was based on measured wall strains. We analyzed the method's ability to estimate the material parameters by generating a set of synthetic strain data by simulating the LV inflation phase with known material parameters. In this way we were able to verify the correctness of the solution and to analyze the effects of measurement and model error on the solution accuracy and stability. A sensitivity analysis was performed to investigate the observability of the material parameters and to determine which parameters to estimate. The results showed a high degree of coupling between the parameters governing the stiffness in each direction. Thus, only one parameter in each of the three directions was estimated. For the tested magnitudes of added noise and introduced model errors, the resulting estimated stress-strain characteristics in the fiber and sheet dircetions converged with good accuracy to the known relationship. The sheet-normal stressstrain relationship had a higher degree of uncertainty as more noise was added and model error was introduced.

\section{Introduction}

Finite element based continuum models of the heart, incorporating a detailed description of myocardial structure, are now frequently used to calculate stress and strain distributions. The major limitation of the predictive accuracy of these models is uncertainties about the best functional form of the constitutive law and the lack of knowledge of the specific law parameters.

In vitro stress-strain testing of myocardial tissue has been used to determine material parameters in the constitutive law (Nielsen et al., 1991; Dokos et al., 2000, 2002). The results from in vitro tests, however, may be questionable as the tissue is disrupted and may be damaged. From human subjects it is not feasible to obtain

*Department of Circulation and Medical Imaging, Norwegian University of Science and Technology, Trondheim, Norway.

$\dagger$ Bioengineering Institute, University of Auckland, New Zealand.

IInstitute for Surgical Research and Department of Cardiology, Rikshospitalet University Hospital, Oslo, Norway.

*Reprinted from Journal of Biomechanics, Vol. 37, Remme et al., 'Development of an in vivo method for determining inaterial properties of passive myocardium', pp. 669-678, (C) 2004 with permission from Elsevier. 
tissue samples. Moulton et al. $(1995,1996)$ developed an in vivo method for determining material properties by fitting a $2 \mathrm{D} \mathrm{FE}$ model to strain measurements from magnetic resonance imaging (MRI) tagging. They were able to estimate the two material parameters of an isotropic, nonlinear, small deformation 2D FE model.

Detailed investigations of the myocardial structure (LeGrice et al., 1997; Smaill and Hunter, 1991) have revealed that the myocardial fibers are arranged in layers or sheets. Based on in vitro biaxial stress-strain experiments (Nielsen et al., 1991), Hunter (1995) proposed an orthotropic 'pole-zero' constitutive law describing the different stress-strain relationships in the fiber, sheet and sheet-normal directions. A 3D FE model of the LV myocardium that includes a description of the morphological fiber and sheet structures and uses the pole-zero constitutive law based on finite deformation theory will give more realistic deformation patterns than the model of Moulton $e t$ al. $(1995,1996)$. However, the 'pole-zero' constitutive law contains 18 material parameters but some are coupled via a micro-structural model (Nash and Hunter, 2001). A parameter estimation technique depends on the observability of the model parameters. If a perturbation of one parameter causes a similar model response to the perturbation of another parameter, then there might be poor observability, which makes the parameter estimation difficult. Stevens et al. (2003) presented a sensitivity analysis of the material parameters that may be used to assess the observability.

The aims of this study were (1) to present a method for estimation of passive myocardial material parameters using the 3D FE model of Costa et al. (1996) and Nash and Hunter (2001), which is based on finite deformation theory and includes the myocardial fiber and sheet orientation and an orthotropic constitutive law and (2) to investigate the feasibility of such a method by performing an observability analysis of the model parameters and checking the solution accuracy and stability with respect to introduced measurement and model error.

The final goal of a material estimation procedure like this is to combine the $3 \mathrm{D}$ FE model with strain measurements from for example MRI tagging. However, to investigate the feasibility of the method it is not necessary to include real strain measurements from MRI tagging. It is better to generate a synthetic test data set from a simulated inflation of the 3D FE model with known material parameters. By applying the material estimation procedure on the synthetic test data we can verify the correctness of the solution, investigate estimation stability and assess the effects of measurement and model errors. For the generation of the test data, the geometry of the FE model should be based on physiological measurements of the inner and outer surfaces of the heart. In this study the inner and outer surfaces were measured with 3D ultrasound.

\section{Methods}

\section{Measurements}

3D ultrasound measurements from four open-chest adult mongrel dogs were used to generate the test data. The study was approved by the National Animal Experimentation Board. Simultaneous measurements of the LV geometry and blood pressure were obtained. The pressure was measured using a $5 \mathrm{~F}$ micromanometertipped catheter (model MPC-500, Millar Instruments, Houston, TX, USA) positioned in the LV. A Vivid FiVe ultrasound scanner (GE Vingmed Ultrasound) with a 3.5MHz probe was used for the imaging. This system enables 3D/4D data acquisition based on a collection of $2 \mathrm{D}$ images. The probe is placed in a rotation adapter that 
rotates the probe $180^{\circ}$ around the central axis of the imaging plane resulting in a conical data set. Apical 2D images were obtained over one heart cycle from 15 scanplanes distributed over the $180^{\circ}$ rotation. The image file was later imported to a software program, EchoPac-3D (GE Vingmed Ultrasound), for post-processing. In this program contours were manually drawn around the endocardium and epicardium for all 15 scan-planes at the time of minimum LV pressure, which approximately corresponds to the beginning of the diastases.

\section{The Finite Element method}

An initial finite element mesh was fitted to the measurcd geometry as described in Hunter et al. (1992) using the prolate spheroidal coordinate system shown in Figure 1.

Investigations of the myocardial structure (LeGrice et al., 1997; Smaill and Hunter, 1991) have revealed that the myocardial fibers are arranged in layers or sheets with three distinct material axes: one along the fiber direction, one orthogonal to the fiber direction but in the sheet and one normal to the sheet. Following LeGrice et al. (2001); the fiber angle was set to vary linearly from $85^{\circ}$ at the endocardial surface to $-60^{\circ}$ at the epicardial surface. For the $L V$ free-wall the sheet angle was set to vary linearly from $-45^{\circ}$ at the endocardial surface to $+45^{\circ}$ at the epicardial surface with respect to the radial coordinate. For the septum the sheet angle was set to vary linearly from $+45^{\circ}$ at the $\mathrm{LV}$ side to $-45^{\circ}$ on the RV side. The imbrication angle (the out-of-plane angle) of the fibers was set to zero.

In vitro biaxial stress-strain experiments on myocardial tissue samples have shown that the stress-strain behavior along the fiber, sheet and sheet-normal directions is

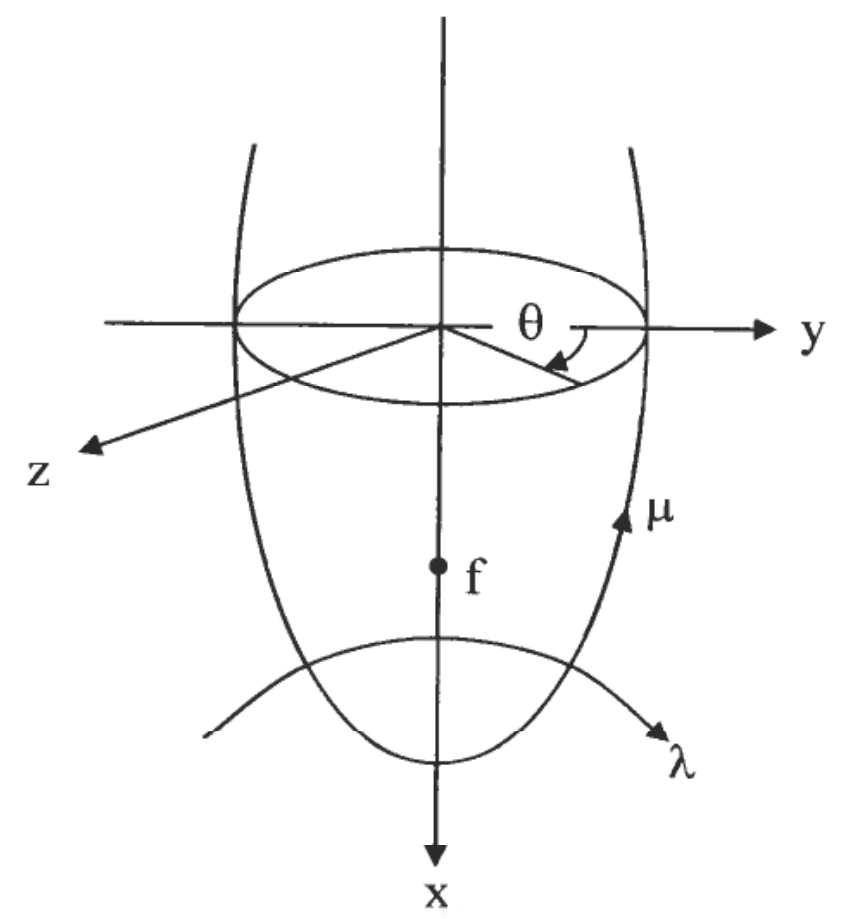

Figure 1. Prolate spheroidal coordinates: $\theta$-circumferential, $\mu$-longitudinal, $\lambda$-transmural, and focus $f$. 
quite different (Smaill and Hunter, 1991). On the basis of these experiments Hunter (1995) proposed a strain energy function called the 'pole-zero' law, equation (1), to describe the stress-strain relationship in the myocardium.

$$
\begin{aligned}
W= & k_{1} \frac{e_{11}^{2}}{\left(a_{1}-e_{11}\right)^{b_{1}}}+k_{2} \frac{e_{22}^{2}}{\left(a_{2}-e_{22}\right)^{b_{2}}}+k_{3} \frac{e_{33}^{2}}{\left(a_{3}-e_{33}\right)^{b_{3}}} \\
& +k_{4} \frac{e_{12}^{2}}{\left(a_{4}-e_{12}\right)^{b_{4}}}+k_{5} \frac{e_{13}^{2}}{\left(a_{5}-e_{13}\right)^{b_{5}}}+k_{6} \frac{e_{23}^{2}}{\left(a_{6}-e_{23}\right)^{b_{6}}}
\end{aligned}
$$

In equation (1) index 1 refers to the fiber direction, index 2 refers to the sheet direction and index 3 refers to the sheet-normal direction. $e_{i j}$ are the covariant components of Green's strain tensor referred to these axes. (Note that these are physical components of strain because they are referred at each point to normalized orthogonal rectangular coordinates.) Indexes 11, 22 and 33 refer to longitudinal strains in the respective directions. The shear terms have indexes 12, 13 and 23. There are three different types of parameters in the constitutive equation: $a_{i}$ are the poles or the limiting strains, $b_{i}$ are related to the curvature of the uniaxial stressstrain relationships and $k_{i}$ are the relative weights of each term to the total strain energy. The components of the 2nd Piola-Kirchhoff stress tensor are derived from the strain energy function in equation (2) for the assumed incompressible material. $p$ is the hydrostatic pressure and $C$ is the Cauchy-Green deformation tensor.

$$
T^{i j}=-p\left(C^{-1}\right)^{i j}+\frac{1}{2}\left(\frac{\partial W}{\partial e_{i j}}+\frac{\partial W}{\partial e_{i j}}\right)
$$

Note that, in the terminology of Nash and Hunter (2001), the $C^{-1}$ term is identical to the contravariant components $a^{i j}$ of the deformed metric tensor for the material coordinates and the strain components are given by $e_{i j}=\frac{1}{2}\left(a_{i j}-A_{i j}\right)$, where $a_{i j}$ and $A_{i j}$ are the covariant components of the deformed and undeformed metric tensors, respectively.

Figure 2 shows the three axial stress-strain curves for the pole-zero law using the parameters in Table 1. The stress-strain relationships in the three directions are clearly different: it is stiffest in the fiber direction, less stiff in the sheet direction normal to the fibers and it is relatively much softer in the direction normal to the sheets. For negative strains, linear stress-strain relationships were used where the slopes for the negative strains were equal to the slopes of the pole-zero law at zero strain.

Nash and Hunter (2001) showed that the shcar poles in equation (1) were related to the axial poles as shown in equation (3). The other 6 shear parameters where set to constant values, leaving the 9 axial parameters to be estimated.

$$
a_{i}=\frac{2 a_{j}}{\sqrt{1+2 a_{j}}}, \text { when } \begin{aligned}
i=4, & a_{j}=\min \left(a_{1}, a_{2}\right) \\
i & =6, a_{j}=\min \left(a_{1}, a_{3}\right)
\end{aligned}
$$

Residual strains (Omens and Fung, 1990) in the fiber direction were included in the reference configuration using the method of Rodriguez et al. (1994). The residual fiber strains varied linearly with a $5 \%$ stretch at the epicardium and a $2 \%$ contraction at the endocardium (Costa et al., 1997). We assumed that the myocardium was in its most stress-free state at the beginning of the diastasis. Thus, the geometric reference 


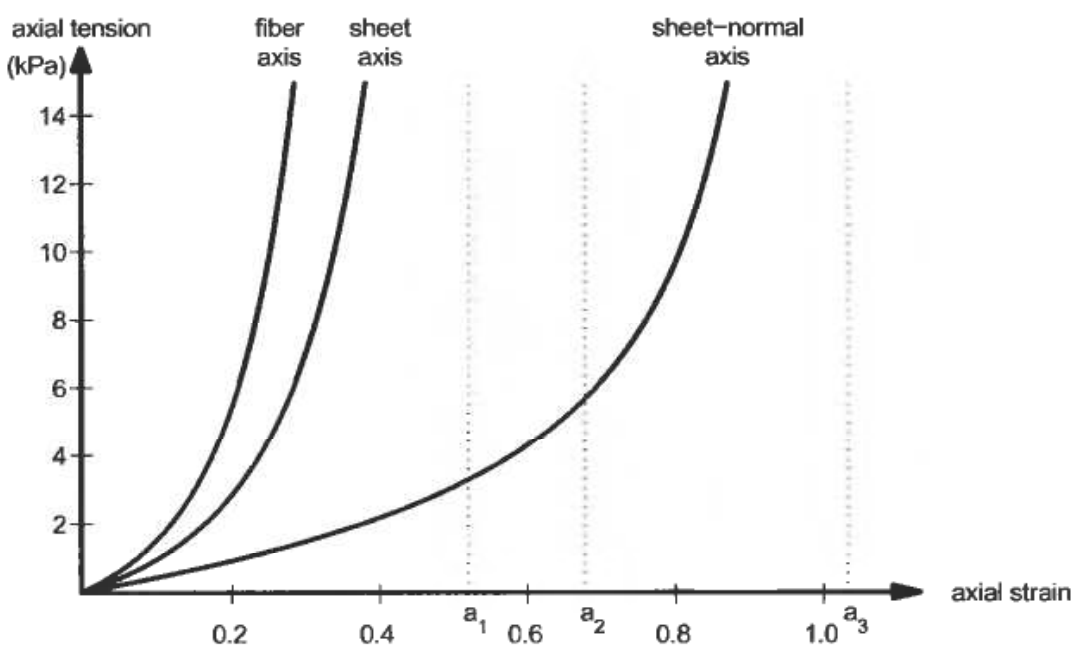

Figure 2. Stress-strain relationships calculated from the pole-zero law (equation 1) using the parameters in Table 1 . The asymptotes on the strain axis are the poles $\left(a_{1}, a_{2}, a_{3}\right)$ representing the elastic strain limits.

Table 1. Material parameters used in the model. The parameter values in this table are referred to as the "true parameter values' in the text.

\begin{tabular}{lcccc}
\hline Type & \multicolumn{2}{c}{ Axial properties } & \multicolumn{2}{c}{ Shear properties } \\
\hline Coefficients & $k_{1}$ & $2.0 \mathrm{kPa}$ & $k_{4}$ & $1.0 \mathrm{kPa}$ \\
& $k_{2}$ & $2.0 \mathrm{kPa}$ & $k_{5}$ & $1.0 \mathrm{kPa}$ \\
& $k_{3}$ & $2.0 \mathrm{kPa}$ & $k_{6}$ & $1.0 \mathrm{kPa}$ \\
Poles & $a_{1}$ & 0.523 & $a_{4}$ & 0.731 \\
& $a_{2}$ & 0.681 & $a_{5}$ & 0.731 \\
& $a_{3}$ & 1.037 & $a_{6}$ & 0.886 \\
Curvatures & $b_{1}$ & 1.351 & $b_{4}$ & 2.0 \\
& $b_{2}$ & 1.351 & $b_{5}$ & 2.0 \\
& $b_{3}$ & 0.398 & $b_{6}$ & 2.0 \\
\hline
\end{tabular}

configuration was fitted to the contours drawn in the ultrasound images at the beginning of the diastasis.

During the inflation of the $\mathrm{LV}$ certain boundary conditions were imposed on the FE mesh. The epicardial apex node was fixed in the $\mu$ and $\theta$ directions. The base and apical nodes where fixed in the $\mu$ direction and the epicardial base nodes were fixed in the $\theta$ direction.

\section{Generation of test data}

In this study we wanted to develop and check the feasibility of a method for determining the stress-strain characteristic of passive myocardium. In order to analyze the method's ability to estimate the material parameters we generated a set of synthetic strain data (in the following called the 'test data') by simulating the LV inflation phase with known material parameters (called the 'true parameters') listed 
in Table 1 (Stevens et al., 2003). By applying the material estimation procedure on the synthetic test data we can verify the solution, investigate estimation stability and assess the effects of measurement and model errors.

To generate the test data we averaged the node coordinates of the geometric FE models of the four dog hearts. With the boundary conditions and the residual strains set, a passive inflation of the LV was simulated. This was done by applying the measured pressure difference of $0.4 \mathrm{kPa}$ (average of the four dogs) between beginning of diastasis and end diastole on the endocardial element-surfaces of the LV. The simulated inflation was initially done with the true parameters. Circumferential, longitudinal and radial wall strains, or rather the extension ratios, $\lambda_{i}$, were written out and saved as the test data at 240 points around the LV ( 20 points circumferentially, 12 longitudinally and 1 radially), giving a total of $N=720$ extension ratio samples. In a clinical study the generated test data would be replaced by measured strains from, for example, MRI tagging.

\section{Parameter estimation}

The estimation procedure was started with an initial guess of the parameter values different from the true parameter values. An iterative estimation process updated the material parameters as a function of the difference between the calculated extension ratios and the test data. When the solution had converged, the iteration process was stopped. The result after the final iteration contained an estimate of the material parameters. A block diagram of the algorithm is shown in Figure 3.

The material parameters, $\alpha$, were estimated by minimizing the least-squares difference between the extension ratios, $\lambda_{i}$, of the test data and the extension ratios, $\hat{\lambda}_{i}$, of the model response:

$$
\left.J(\alpha)=\frac{1}{2}[\hat{\Lambda} \alpha)-\Lambda\right]^{T}[\hat{\Lambda}(\alpha)-\Lambda]
$$

Model response: Test date: Parameters:

$$
\hat{\Lambda}(\alpha)=\left[\begin{array}{c}
\hat{\lambda}_{1} \\
\hat{\lambda}_{2} \\
\vdots \\
\hat{\lambda}_{720}
\end{array}\right] \quad \boldsymbol{\Lambda}=\left[\begin{array}{c}
\lambda_{1} \\
\lambda_{2} \\
\vdots \\
\lambda_{720}
\end{array}\right] \quad \alpha=\left[\begin{array}{c}
k_{1} \\
a_{1} \\
b_{1} \\
\vdots \\
b_{3}
\end{array}\right]=\left[\begin{array}{c}
\alpha_{1} \\
\alpha_{2} \\
\alpha_{3} \\
\vdots \\
\alpha_{9}
\end{array}\right]
$$

An improved estimate of $\alpha$ is obtained by adding an increment $h$ that decreases the error of the model response. The increment $h$ is found as shown in equations (6) and (7) by using the Gauss-Newton method to minimize the least-squares function in equation (4).

$$
\begin{gathered}
\mathbf{h}=\left(S^{T} S\right)^{-1} S^{T}(\boldsymbol{\Lambda}-\hat{\Lambda}(\alpha)) \\
S=\frac{\partial \hat{\Lambda}}{\partial \alpha}=\left[\begin{array}{cccc}
\frac{\partial \hat{\lambda}_{1}}{\partial \alpha_{1}} & \frac{\partial \hat{\lambda}_{1}}{\partial \alpha_{2}} & \cdots & \frac{\partial \hat{\lambda}_{1}}{\partial \alpha_{n}} \\
\frac{\partial \hat{\lambda}_{2}}{\partial \alpha_{1}} & \frac{\partial \hat{\lambda}_{2}}{\partial \alpha_{2}} & \cdots & \frac{\partial \hat{\lambda}_{2}}{\partial \alpha_{n}} \\
\vdots & \vdots & \ddots & \vdots \\
\frac{\partial \hat{\lambda}_{N}}{\partial \alpha_{1}} & \frac{\partial \hat{\lambda}_{N}}{\partial \alpha_{2}} & \cdots & \frac{\partial \hat{\lambda}_{N}}{\partial \alpha_{n}}
\end{array}\right]
\end{gathered}
$$




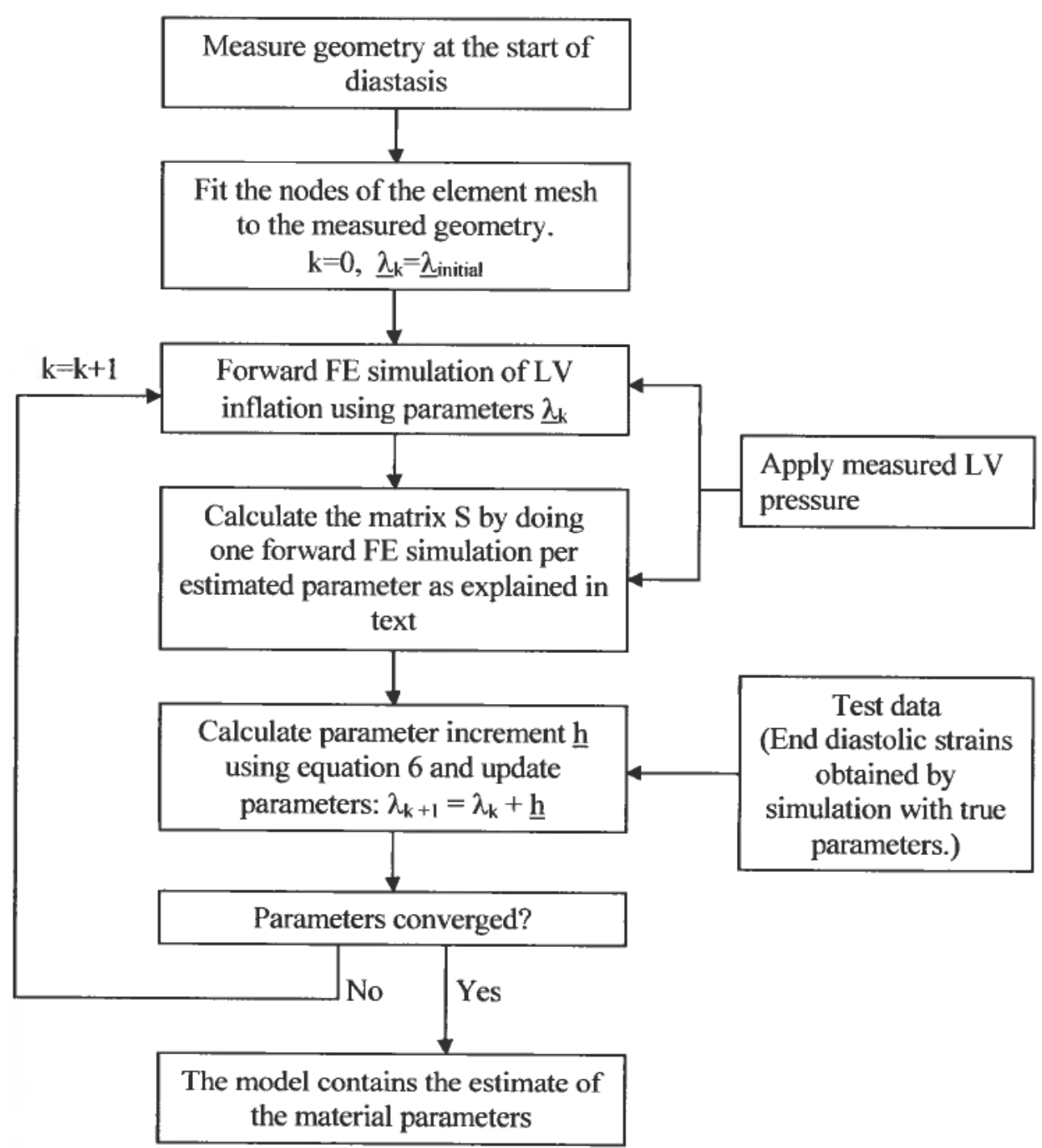

Figure 3. Flow diagram of the material property estimation procedure.

In equation (7) $N$ is the number of extension ratios and $\mathrm{n}$ is the number of parameters. The matrix $S$ was calculated at each iteration of the feedback loop in the blockdiagram in Figure 3. A finite difference method was used to calculate the column vectors in $S$ : First, the simulation result using the parameters $\alpha_{k}$ was entered in the column vector $\hat{\Lambda}_{0}$. Then, parameter $m$ in $\alpha_{k}$ was increased by $10 \%$ while the other parameters were kept the same as in the original $\alpha_{k}$. The FE simulation was run and the result written into column vector $\hat{\Lambda}_{m}$. Column $m$ of $S$ was calculated as $\left(\hat{\Lambda}_{m}-\hat{\Lambda}_{0}\right) / \Delta \alpha_{m}$. When all the columns in $S$ were calculated, the parameter increment h was found as shown in equation (6). The new parameters were found using $\boldsymbol{\alpha}_{k+1}=\boldsymbol{\alpha}_{k}+\mathbf{h}$. If the components of the incremental vector $\mathbf{h}$ were below certain thresholds the itcration process was stopped as the parameters were considered to have converged. 


\section{Sensitivity analysis}

An analysis of the method's ability to estimate the parameters was performed. The matrix $S$ expresses the model response sensitivity to variations of the material parameters. If the variation of one parameter causes a similar response as the variation of another, the estimation process might be ambiguous. This will result in two almost dependent column vectors in $S$ for the corresponding parameters. To analyze the correlation between the various parameters' influence on the modelresponse each column in $S$ was normalized creating the matrix $s$ and hence the sensitivity matrix $s^{T} S$, in which a value close to \pm 1 for element $e_{i j}$ means the parameter $i$ and $j$ are highly correlated. A high correlation may result in a nonconverging estimation problem. A solution is then to put one of two correlated parameters to a constant value and estimate only one of them.

If a variation of one parameter makes little difference on the model response, it will be difficult to estimate this parameter and the parameter's influence on the deformation may be minor. We compared the sensitivity of the model response to a variation in each parameter by looking at the norm of the column vector $\left(\hat{\mathbf{\Lambda}}_{m}-\hat{\mathbf{\Lambda}}_{0}\right)$.

It was considered unlikely that the estimation procedure could estimate all 9 parameters unambiguously. The above analysis helped us rule out several parameters and left us with three parameters to estimate - one in each of the axial directions. These three parameters were used in the further analysis of the method.

Several tests were performed to check the convergence of the estimates of the three parameters (the other parameters were set to constant values). First the three parameters were estimated while the others were set to their correct (known) values. Then, the estimation was carried out while the other parameters were set to incorrect values, making the material either stiffer or softer than the true material. To make the material stiffer the constant $k$ 's and $b$ 's were increased with $20 \%$ while the poles were decreased by $20 \%$. To make it softer the opposite changes were made. Note that the value of the denominator is most often below 1 in the terms of equation (1). Thus, an increase of the exponential $b$ will increase the energy of the term and make it stiffer. Figure 4a shows stress-strain curves in the fiber direction with different parameter sets. The solid curve is calculated with the true parameter values $\left(k_{1}=2.0\right.$, $\left.a_{1}=0.523, b_{1}=1.351\right)$. The dashed curve is the initial stiffer guess where the parameters are changed by $20 \%\left(k_{1}=2.4, a_{1}=0.4184, b_{1}=1.6212\right)$.

The next test was to add noise to the test data, $\lambda_{i}+w_{i}$ and check the convergence of the estimated parameters. The convergence was examined for different levels of noise $w_{i}\left(w_{i}=\right.$ random numbers uniformly distributed in the intervals): $w_{i}=0$ (no noise), $w_{i} \in[-0.01,0.01], w_{i} \in[-0.05,0.05]$ and $w_{i} \in[-0.1,0.1]$.

The last test was both to add noise to the test data and to set the other parameters to constant incorrect values and then run the estimation procedure.

\section{Results}

The analysis of the sensitivity matrices resulting from the estimation of all 9 parameters showed a high correlation between several of the parameters. There was an obvious pattern to the correlation. The three parameters $k_{1}, a_{1}$ and $b_{1}$ were highly correlated with each other. So were $k_{2}, a_{2}$ and $b_{2}$. Table 2 shows an average of two sensitivity matrices: one where the parameter increment was $+10 \%$ to calculate the $S$ matrix and one where the increment was $-10 \%$. The model response had substantially higher sensitivity to variations of the parameters in the fiber and sheet 
Table 2. Sensitivity matrix. Average result of two sensitivity matrices: one where the parameter increment was $+10 \%$ and one with a $-10 \%$ increment. The values are a reflection of the correlation between two parameters, i.e. a value close to 1 , means a change in one parameter has a similar effect on the model output to a change of the other parameter.

\begin{tabular}{lrrrrrrrrr}
\hline & $k_{1}$ & $a_{1}$ & \multicolumn{1}{c}{$b_{1}$} & \multicolumn{1}{c}{$k_{2}$} & \multicolumn{1}{c}{$a_{2}$} & \multicolumn{1}{c}{$b_{2}$} & \multicolumn{1}{c}{$k_{3}$} & \multicolumn{1}{c}{$a_{3}$} & $b_{3}$ \\
\hline$k_{1}$ & 1.00 & -0.99 & 0.99 & 0.77 & -0.87 & 0.78 & 0.32 & -0.50 & 0.34 \\
$a_{1}$ & -0.99 & 1.00 & -0.99 & -0.75 & 0.86 & -0.75 & -0.32 & 0.53 & -0.37 \\
$b_{1}$ & 0.99 & -0.99 & 1.00 & 0.77 & -0.86 & 0.77 & 0.31 & -0.50 & 0.33 \\
$k_{2}$ & 0.77 & -0.75 & 0.77 & 1.00 & -0.93 & 0.99 & 0.43 & -0.19 & 0.49 \\
$a_{2}$ & -0.87 & 0.86 & -0.86 & -0.93 & 1.00 & -0.93 & -0.55 & 0.53 & -0.39 \\
$b_{2}$ & 0.78 & -0.75 & 0.77 & 0.99 & -0.93 & 1.00 & 0.43 & -0.19 & 0.49 \\
$k_{3}$ & 0.32 & -0.32 & 0.31 & 0.43 & -0.55 & 0.43 & 1.00 & -0.59 & 0.27 \\
$a_{3}$ & -0.50 & 0.53 & -0.50 & -0.19 & 0.53 & -0.19 & -0.59 & 1.00 & 0.01 \\
$b_{3}$ & 0.34 & -0.37 & 0.33 & 0.49 & -0.39 & 0.49 & 0.27 & 0.01 & 1.00 \\
\hline
\end{tabular}

Table 3. Sensitivity values. Average of two sensitivity values for each parameter: one where the parameter increment was $+10 \%$ and one with a $-10 \%$ increment. Each value is calculated as the norm of the vector that contains the difference at each measurement point between the model response with one perturbed parameter $( \pm 10 \%)$ and the model response simulated with the true parameter values.

\begin{tabular}{ccccccccc}
\hline$k_{1}$ & $a_{1}$ & $b_{1}$ & $k_{2}$ & $a_{2}$ & $b_{2}$ & $k_{3}$ & $a_{3}$ & $b_{3}$ \\
\hline 0.04406 & 0.07057 & 0.04785 & 0.04224 & 0.06361 & 0.02185 & 0.00918 & 0.01293 & 0.00013 \\
\hline
\end{tabular}

directions than in the sheet-normal direction (Table 3). The highest sensitivities within the three directions were for changes of the pole parameters $\left(a_{1}, a_{2}\right.$ and $\left.a_{3}\right)$.

From the results of the sensitivity analysis it was decided to estimate only one parameter in each direction. It was observed that a change of either the pole ' $a$ ' or the curvature parameter ' $b$ ' could compensate better for wrong values in the two other parameters than the coefficient parameter ' $k$ ' (see Figure 4a). From these results we chose to estimate the poles $a_{1}, a_{2}$ and $a_{3}$.

The first trial was to estimate the three chosen parameters with the other constant parameters set to their true values. Initially, the estimated parameters were set $\pm 20 \%$ off their true values. With no added noise $a_{1}$ and $a_{2}$ had practically converged to their true values after a single iteration. The same was the case for $a_{3}$ after two iterations. Table 4 summarizes the estimation results after three iterations. Figure $5 \mathrm{a}-\mathrm{c}$ shows the convergence of the parameter $a_{1}, a_{2}$ and $a_{3}$, respectively. Initial values of $\pm 50 \%$ and $\pm 75 \%$ off their true values were tested. A change of $50 \%$ of the pole value gives a substantial shift of its stress-strain curve. All 8 combinations of setting: $a_{1}, a_{2}$ and $a_{3}$ too low; $a_{1}, a_{2}$ too low and $a_{3}$ too high; etc; to the last combination of $a_{1}, a_{2}$ and $a_{3}$ too high, were also tested. These tests converged as well, but needed $1-4$ more iterations in some cases.

As shown in Figure 5 the fiber pole, $a_{1}$, converged quickly and with just a small offset $(< \pm 2.5 \% \mathrm{off})$ from its true value for all three levels of noise added to the test data. The sheet pole parameters, $a_{2}$, converged quickly and with small offset $(< \pm 2.5 \%$ off) from its true value for the two lowest levels of noise, for the highest noise level it ended up on a value $13 \%$ higher than its true value. The sheet-normal pole, $a_{3}$, ended up with offsets of $6 \%, 18 \%$ and $-33 \%$ for the three levels of noise added, respectively. 


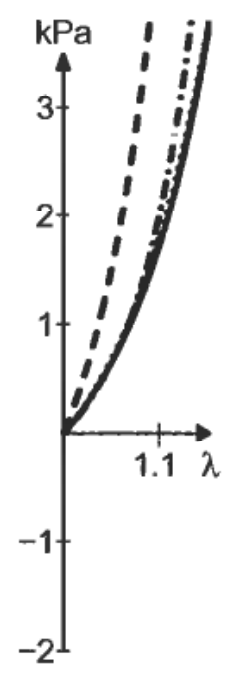

(a)

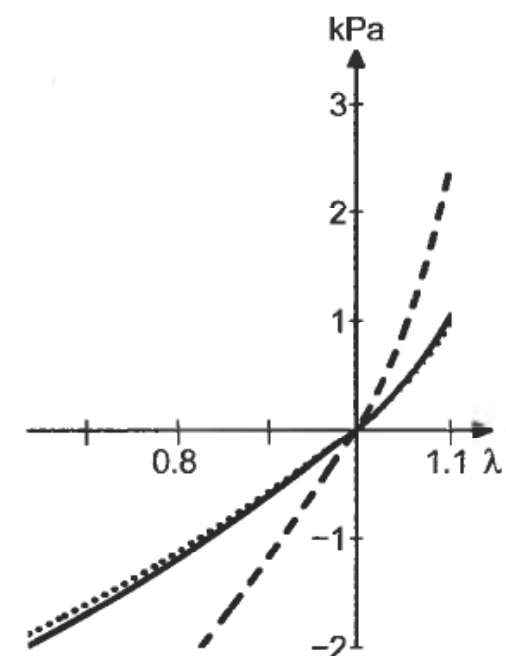

(b)

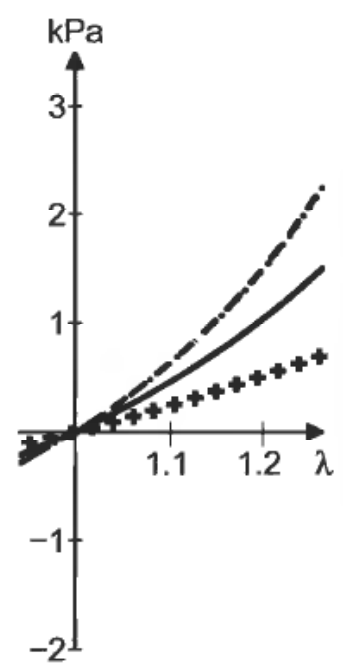

(c)

Figure 4. Stress-stretch relationship in fiber (a), sheet (b), and sheet-normal (c) directions. The solid lines are the relationships using the true parameter values in table 1 . The dashed lines are the relationships when each of the parameters is changed to a $20 \%$ stiffer value. The dotted lines are the result after the estimation procedure $\left(a_{1}=0.63, a_{2}=0.84, a_{3}=0.83\right)$. The dash-dotted line in (a) is when $k_{1}(=1.06)$ is estimated instead of $a_{1}$. The + line in (c) is the result of $a_{3}=3.29$ in the case when the constant parameters are set $20 \%$ softer. Note that the dotted line in (a) almost overlaps the solid line, while in (c) the dotted line almost overlaps the dashed line. The abscissas are the ranges of fiber, sheet, and sheet-normal stretches. The minimum and maximum stretch range for each of the three plots is obtained by finding the minimum and maximum stretch of all the LV Gauss points in each respective direction of the simulation result.

Table 4. Estimation results. The values after three iterations of the estimated poles $a_{1}, a_{2}$, and $a_{3}$ with different ranges of noise and different values of the constant $k$ and $b$ parameters. For the value marked with *, see text.

\begin{tabular}{|c|c|c|c|c|c|c|c|c|c|c|c|c|}
\hline \multirow{3}{*}{$\begin{array}{l}\text { noise } \\
a_{1}\end{array}$} & \multicolumn{4}{|c|}{ Correct $k$ 's and $b$ 's } & \multicolumn{4}{|c|}{$\begin{array}{l}\text { Too stiff } k \text { 's and } b \text { 's } \\
1.2 \times k_{i \text { true }}, 1.2 \times b_{i} \text { true }\end{array}$} & \multicolumn{4}{|c|}{$\begin{array}{l}\text { Too soft } k \text { 's and } b \text { 's } \\
0.8 \times k_{i \text { true }}, 0.8 \times b_{i \text { true }}\end{array}$} \\
\hline & 0 & {$[ \pm 0.01]$} & \pm 0.05 & {$[ \pm 0.10]$} & 0 & {$[ \pm 0.01$} & \pm 0.0 & {$[ \pm 0.10]$} & 0 & {$[ \pm 0.01$} & \pm 0.05 & {$[ \pm 0.10]$} \\
\hline & 0.52 & 0.53 & 0.53 & 0.52 & 0.63 & 0.64 & 0.64 & 0.65 & 0.41 & 0.41 & 0.40 & 0.37 \\
\hline$a_{2}$ & 0.68 & 0.67 & 0.67 & 0.77 & 0.84 & 0.83 & 0.83 & 0.91 & 0.47 & 0.46 & 0.48 & 0.57 \\
\hline$a_{3}$ & 1.04 & 1.10 & 1.22 & 0.70 & 0.83 & 0.86 & 0.92 & $0.61^{*}$ & 3.29 & 5.06 & 4.82 & 1.09 \\
\hline
\end{tabular}

Figure 6 shows the convergence of the parameters when all the parameters were changed $20 \%$ in the stiffer direction (dashed lines) and 20\% in the softer direction (dotted lines). The estimation results are summarized in Table 4. In general the estimated values of $a_{1}$ and $a_{2}$ converged to values that compensated for the incorrect constant parameter values of the k's and b's as can be seen in the case of initial too stiff parameters in Figure 4. The sheet-normal pole, $a_{3}$, converged to a paradoxical value, i.e. it got even more stiff or soft when $k_{3}$ and $b_{3}$ were set too stiff or soft, respectively. The convergence pattern for $a_{3}$ was also quite oscillatory. In the case of too stiff $k_{3}$ and $h_{3}$ and the highest noise level, the value of $a_{3}$ was -0.35 after the first iteration. An automatic correction routine in the estimation code changed the 


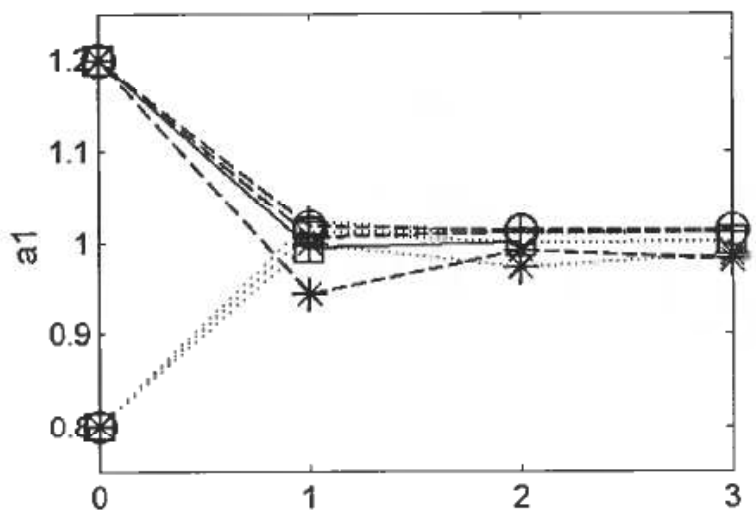

(a)

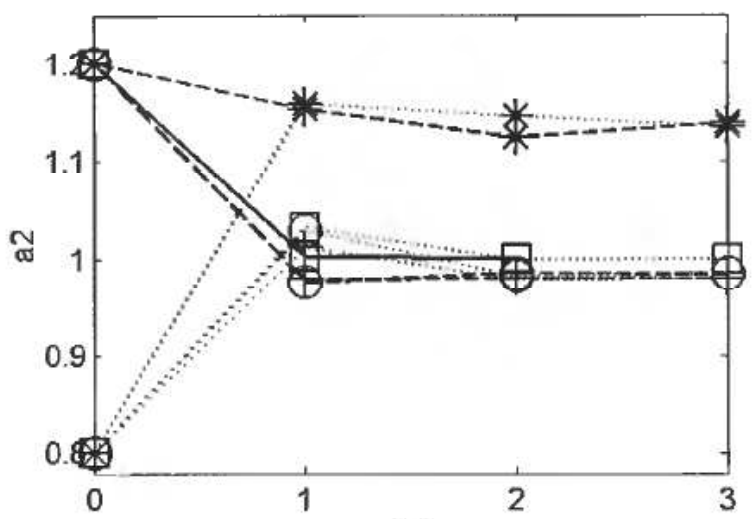

(b)

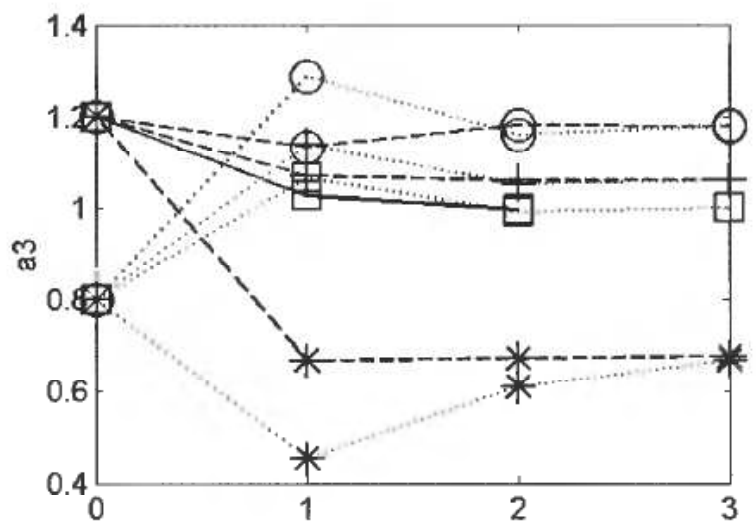

(c)

Number of iterations

Figure 5. Convergence of parameters $a_{1}, a_{2}$, and $a_{3}$ in (a), (b), and (c), respectively. The plots are normalized with respect to their true parameter values. The convergence of the parameters with different levels of noise added to the measurements is shown with different line-markers; $\square: w_{i}=0$ (no noise), $+: w_{i} \in[-0.01,0.01], 0: w_{i} \in[-0.05,0.05]$, and $*: w_{i} \in[-0.1,0.1]$. The initial values were offset by $+20 \%$ (dashed lines) and $-20 \%$ (dotted lines) from their true parameter values. 
unphysiological value and replaced it with half its previous value $(0.4148)$ as shown in Figure 6. From then on the parameter converged.

The computation time needed for the estimation depended on the number of estimated parameters. One forward FE simulation required approximately 80 seconds using 15 CPUs on an Origin 3800L SGI machine with $600 \mathrm{MHz}$ R14k processors. For every iteration in the estimation routine, one FE simulation was done for each estimated parameter plus one with the updated parameter set, i.e. four FE simulations for every iteration when three parameters were estimated.

\section{Discussion}

The finite element method is a powerful tool to calculate strain and stress distributions in loaded structures with a complex geometry, but for accurate stress calculations the material properties need to be known. In this study we have presented a model-based estimation procedure where we have applied the FE method to characterize the stress-strain relationship in passive LV myocardium. We have used a 3D FE model of the LV myocardium that includes finite deformation theory, morphological fiber and sheet structure and a non-linear orthotropic constitutive law. The estimation procedure is based on measured strains (from for example tagged MRI) where the measured strains are compared with the model-predicted strains and the errors between the two are minimized in a least-squares sense by optimizing the material parameters in the model.

The investigation of the parameter observability showed a high degree of coupling between some of the parameters. The three parameters in the fiber direction were highly correlated; so were the three parameters in the sheet direction as seen in Table 2. The correlation may be explained by the similar response of the stress-strain curve when the parameters are varied. An increase in $k$ and $b$ both raise the curve and vice versa. An increase of the pole parameter, $a$, lowers the stiffness curve and vice versa. Thus, parameters $k$ and $b$ are positively correlated, while they are negatively correlated with parameter a as seen in Table 2 . The close relationship between the parameters is shown in Figure 4. Figure 4a shows the true fiber stiffness curve (solid line), a curve where $k_{1}$ and $b_{1}$ are increased by $20 \%$ and $a_{1}$ is decreased by $20 \%$ to make it stiffer (dashed line). The dotted curve (practically overlapping the solid line) has the same parameters as the stiffer one except $a_{1}$, which has been estimated using the parameter estimation method. The estimation resulted in a $20 \%$ higher value of $a_{1}$ compared to its true value. This increase of $a_{1}$ compensates for the increased stiffness in $k_{1}$ and $b_{1}$ and the resulting stress-strain relationship is almost identical to the true curve. The dash-dotted line shows the similar, but in this case parameter $k_{1}$ is estimated instead of $a_{1}$. The reduction of $k_{1}$ compensates for the increased stiffness in $b_{1}$ and $a_{1}$. The compensation with the pole parameter $a_{1}$ gives a better fit to the true curve than with the scaling parameter $k_{1}$. Outside the range of strain plotted in the figure the curves will diverge but we believe the strains are limited to approximately this range in the phase between the beginning of diastasis and the end of diastole. An important point here is that the pole-zero terms in the strain energy function (1) are designed to represent the full range of elastic strain but the parameters are in fact only being fitted for a limited portion of this range. This means that the curve is over parameterized for these tests and that what is significant is the fitted stressstrain curve, not the individual parameter values. Future tests over wider ranges of strain are needed to better characterize the individual parameter values. 


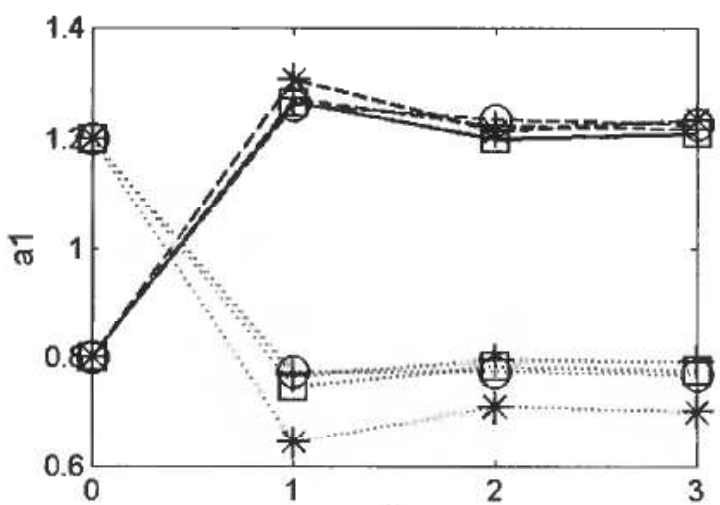

(a)

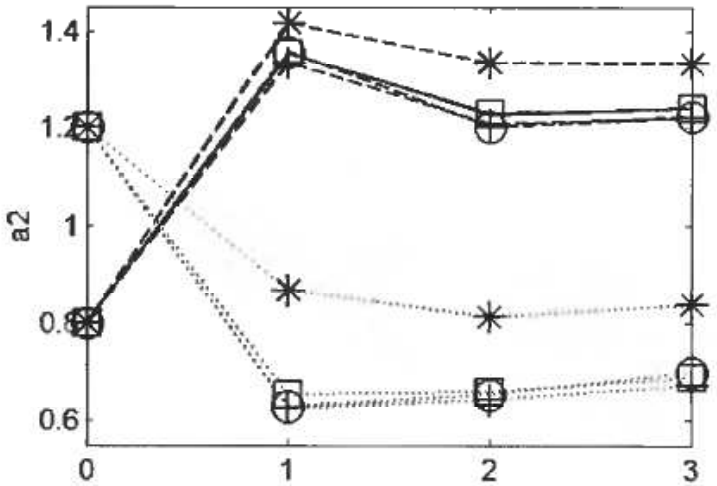

(b)

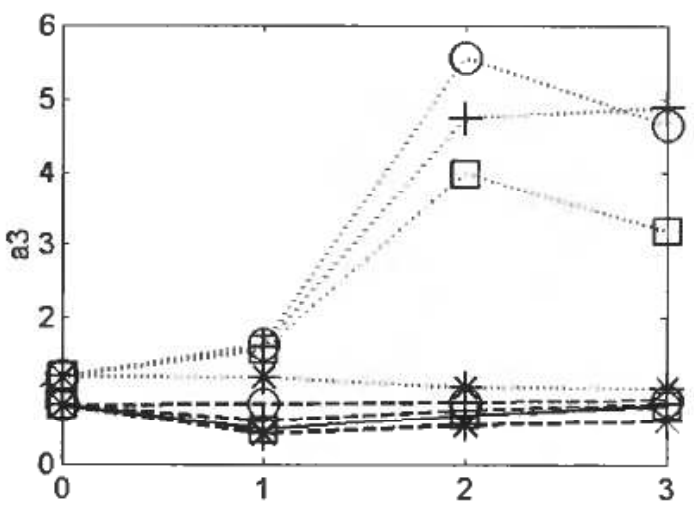

(c)

Number of iterations

Figure 6. Convergence of parameters $a_{1}, a_{2}$, and $a_{3}$ in (a), (b), and (c), respectively, when the values of the constant parameters, the $k$ 's and $b$ 's, are set $20 \%$ too high/stiff (dashed lines) and $-20 \%$ too low/soft (dotted lines). The plots are normalized with respect to their true parameter values. The convergence of the parameters with different levels of noise added to the measurements is shown with different line-markers; $\square: w_{i}=0$ (no noise), $+: w_{i} \in[-0.01,0.01]$, $\mathrm{O}: \mathrm{w}_{i} \in[-0.05,0.05]$, and $*: \mathrm{w}_{i} \in[-0.1,0.1]$. Initially, the start values of the poles are increased $20 \%$ to make the material even softer (dashed lines) or decreased $20 \%$ to make it even stiffer (dotted lines). 
The high degree of coupling between the parameters may make it difficult to estimate all of them and get proper convergence. But from the results showing the compensation of wrong values on two parameters by a change of the third parameter value, we believe it may be possible to estimate the stress-strain characteristics by estimating only one parameter in each direction. The choice falls on the estimation of the pole parameters $a_{1}, a_{2}$ and $a_{3}$ which yield closer fits to the stress-strain curves and give the highest model response sensitivities in their respective directions. The estimation of $a_{1}$ and $a_{2}$ showed good robustness. They converged well for all cases with added noise and wrong values of the other parameters. There was more uncertainty connected to the estimation result of the sheet-normal pole $a_{3}$. With correct values of the $\mathrm{k}$ and $\mathrm{b}$ parameters, $a_{3}$ was estimated with a fair degree of accuracy. The error of the estimate was dependent on the degree of noise in the test data. However, when the constant parameters were set too stiff, $a_{3}$ ended up with a value that made the sheet-normal characteristic even stiffer and vice versa.

There may be different reasons for the poorer estimate of $a_{3}$. The contribution to the total strain energy from the sheet-normal term in equation (1) is the lowest of the axial terms as can be seen in Figures 2 and 4 . Thus the sensitivity of the model output to a variation in a sheet-normal parameter would be lower than for a parameter in the fiber or sheet direction, which is seen in Table 2. It may then be that the fiber and sheet stiffness cause the limiting deformation and a variation of the sheet-normal stiffness is so soft it makes little difference. In this study we have not attempted to estimate any shear parameters. Dokos et al. (2002) conclude that the contribution of shear stiffness to the passive LV deformation is relatively small, so the sensitivity to a change in a shear parameter is likely to be small.

During diastole the $\mathbf{L V}$ is elongated and the wall thins. The fibers are stretched, the myocardial sheets lie primarily in the transmural direction and are mostly compressed, whereas there is mainly a stretch in the sheet-normal direction which is primarily aligned with the base-apex direction. During systole the opposite occurs. Since the sheet-pole governs the limiting stretch of the sheet it may seem more appropriate to estimate this parameter during systole while the sheets are mostly stretched.

In this study we wanted to investigate the feasibility of the estimation procedure and we generated a set of test data from simulations instead of using real strain measurements. This enabled us to evaluate the results against the known solution and to analyze the effects of noise and model error. The results encourage the validation of the method on real measurement data. In a clinical study measured strains from for example tagged magnetic resonance images would replace the generated test data. When the method is used on real measurements some errors may arise that are not apparent in this study.

The boundary conditions on the FE model may be a source of error. The effects of the pericardium, the intersections with the right ventricle (RV) and the RV pressure on the septum are all ignored in the boundary conditions.

Another factor that may influence the results is that the LV is not necessarily in an unloaded state, i.e. the $\mathrm{LV}$ pressure $\neq 0 \mathrm{mmHg}$, at the beginning of diastasis. This pressure will produce stresses and strains that arise in addition to the residual strains and stresses in the unloaded reference state. A better approximation to the initially reference configuration of our FE model may be to first inflate the model from 0 $\mathrm{mmHg}$ to the measured pressure at the beginning of diastasis. Then import the calculated nodal stresses and strains back to the initial geometry and use this as the 
starting configuration of the estimation routine. This approximation assumes that the strains caused by the non-zero pressure are small and that the stress-strain relationship for these small strains is approximately linear.

We have assumed that the fiber and sheet orientations are the same in all hearts and have not looked into the effects of possible variations of the fiber and sheet orientations in different hearts. In regionally diseased hearts such as in the case of ischemia and infarction there are likely to be regional variations of the stiffness of the myocardium. This may even be the case in normal hearts (Novak et al. 1994). We have assumed a homogenous set of material parameters throughout the LV myocardium. A further development of the method may include regionally varying material parameters. Extension of the method to include regions with different material parameters may be done by adding a set of parameters per region to the estimation routine. However, there is likely to be a limit to the number of observable parameters.

\section{Acknowledgement}

This research has been supported in part by The Research Council of Norway grants \#128267/320 and \#139327/300 and through a grant of computing time (Program for supercomputing).

\section{References}

Costa, K. D., Hunter, P. J., Wayne, J. S., Waldman, L. K., Guccione, J. M. \& McCulloch, A. D. (1996). A three-dimensional finite element method for large elastic deformations of ventricular myocardium: Part II-Prolate spherical coordiantes, ASME Journal of Biomechanical Engineering, 118, $464-472$.

Costa, K. D., May-Newman, K., Farr, D., O'Dell, W. G., McCulloch, A. D. \& Omens, J. H. (1997). Three-dimensional residual strain in midanterior canine left ventricle, American Journal of Physiology, 273, H1968-H1976.

Dokos, S., LeGrice, I. J., Smaill, B. H., Kar, J. \& Young, A. A. (2000). A triaxialmeasurement shear-test device for soft biological tissues, ASME Journal of Biomechanical Engineering, 122, 471-478.

Dokos, S., Smaill, B. H., Young, A. A. \& LeGrice, I. J. (2002). Shear properties of passive ventricular myocardium, American Journal of Physiology, 283, H2650-H2659.

HUNTER, P. J. (1995). Myocardial constitutive laws for continuum mechanics models of the heart, Advances in Experimental Medicine and Biology, 382, 303-318.

Hunter, P. J., Nielsen, P. M., Smaill, B. H., LeGrice, I. J. \& Hunter, I. W. (1992). An anatomical heart model with applications to myocardial activation and ventricular mechanics, Critical Reviews in Biomedical Engineering, 20, 403-426.

LeGrice, I. I., Hunter, P. I. \& Smail.t, B. H. (1997). I aminar structure of the heart: a mathematical model, American Journal of Physiology, 272, H2466-H2476.

LeGrice, I., Hunter, P., Young \& A., Smaill, B. (2001). The architecture of the heart: a data-based model, Philosophical Transaction of the Royal Society, 359, 1217-1232.

Moulton, M. J., Creswell, L. L., Actis, R. L., Myers, K. W., Vannier, M. K., Szabo, B. A. \& PASQUE, M. K. (1995). An inverse approach to determining myocardial material properties, Journal of Biomechanics, 28, 935-948.

Moulton, M. J., Creswell, L. L., Downing, S. W., Actis, R. L., Szabo, B. A. \& Pasque, M. K. (1996). Myocardial material property determination in the in vivo heart using magnetic resonance imaging, International Journal of Cardiac Imaging, 12, 153-167.

NASH, M. P. \& Hunter, P. J. (2001). Computational mechanics of the heart, Journal of Elasticity, 61, 113-141.

Nielsen, P. M. F., Hunter, P. J. \& Smaill, B. H. (1991). Biaxial testing of membrane biomaterials: Testing equipment and procedures, ASME Journal of Biomechanical Engineering, 113, 295-300. 
NovaK, V.P, Yin, F. C. \& HuMPHREY, J. D. (1994). Regional mechanical properties of passive myocardium, Journal of Biomechanics, 27, 403-412.

OMEns, J. H. \& FunG, Y. C. (1990). Residual strain in the rat left ventricle, Circulation Research, 66, 37-45.

Rodriguez, E. K., Hoger, A. \& McCulloch, A. D. (1994). Stress-dependent finite growth in soft elastic tissue, Journal of Biomechanics, 27, 455 467.

Smaill, B. H. \& HunTER, P. J. (1991). Structure and function of the diastolic heart: Material properties of passive myocardium. In: Glass, L., Hunter, P. J. \& McGulloch, A. D. (Eds.), Theory of heart: biomechanics, biophysics, and nonlinear dynamics of cardiac function, pp. 1-29 (New York: Springer-Verlag).

Stevens, C., Remme, E., LeGrice, I. \& Hunter, P. (2003). Ventricular Mechanics in diastole: material parameter sensitivity, Journal of Biomechanics, 36, 737-748. 